\title{
EMS Heroin Overdoses with Refusal to Transport \& Impacts on ED Overdose Surveillance
}

\section{Peter Rock, Michael Singleton}

College of Public Health, University of Kentucky, Lexington, Kentucky, United States

Objective

The aim of this project was to explore changing patterns in patient refusal to transport by emergency medical services for classified heroin overdoses and possible implications on heroin overdose surveillance in Kentucky.

\section{Introduction}

As a Centers for Disease Control and Prevention Enhanced State Opioid Overdose Surveillance (ESOOS) funded state, Kentucky started utilizing Emergency Medical Services (EMS) data to increase timeliness of state data on drug overdose events in late 2016. Using developed definitions of heroin overdose for EMS emergency runs, Kentucky analyzed the patterns of refused/transported EMS runs for both statewide and local jurisdictions. Changes in EMS transportation patterns of heroin overdoses can have a dramatic impact on other surveillance systems, such as emergency department (ED) claims data or syndromic surveillance (SyS) data.

\section{Methods}

As part of the ESOOS grant, Kentucky receives all emergency-only EMS runs monthly from Kentucky Board for Emergency Medical Services, Kentucky State Ambulance Reporting System data. Heroin cases were classified based on text and medications (Narcan) administered, with comparisons to historic data discussed elsewhere (Rock \& Singleton, 2018). Transportation classifications are based on EMS standard elements defining treatment with transportation vs refusal to transport to hospital and canceled runs were excluded. Initial analysis included trend analysis at state and local levels, as well as demographic comparisons of refusal vs transported heroin overdose encounters.

\section{Results}

Statewide trends in EMS heroin overdoses with refusal transport significantly increased from 5\% ( $\mathrm{n}=42$ ) in 2016 quarter three to $22 \%(n=290)$ in 2018 quarter two (Fig 1). Initial demographic analysis does not show any significant difference between refusals/transported for age, gender, or race. However, there are significant differences among geographic regions in Kentucky with heroin encounter refusal proportion ranging from 3\%-48\% in 2018 quarter two. Specifically, one urban area (Fig 2) shows the change in proportion of refusal increasing from $15 \%(n=23)$ in 2016 quarter three to $47 \%(n=110)$ in 2018 quarter two. In this geographic area, combined refused/transported EMS heroin overdoses compared to traditional ED data demonstrates opposing heroin overdose patterns for the same local with EMS showing and increasing trend overtime and ED showing a decreasing trend (Fig 3).

\section{Conclusions}

Traditional public health surveillance for heroin overdose has historically relied on ED billing data, though agencies are starting to use syndromic surveillance, too (Vivolo-Kantor et al., 2016). These systems share similar underlying ED data, albeit with different components, quality, and limitations. However, in terms of the overdose epidemic, both are limited to only heroin overdoses that result in ED hospital encounters. The recent drastic increase in refused transport can have significant impacts on heroin surveillance. Jurisdictions relying on SyS or ED data for monitoring overdose patterns and/or evaluating interventions may be significantly underestimating acute overdose occurrence in the population. This analysis highlights the importance of this preclinical data source in surveillance of the heroin epidemic.

\section{Acknowledgement}

We acknowledge and thank the following agencies for their support of this work: The Kentucky Department for Public Health, Kentucky Board of Emergency Medical Services, the Kentucky Office of Health Policy, the National Syndromic Surveillance Program, and the Centers for Disease Control and Prevention. Supported by Cooperative Agreement Number 5NU17CE924880-

ISDS Annual Conference Proceedings 2019. This is an Open Access article distributed under the terms of the Creative Commons AttributionNoncommercial 4.0 Unported License (http://creativecommons.org/licenses/by-nc/3.0/), permitting all non-commercial use, distribution, and reproduction in any medium, provided the original work is properly cited. 
ISDS 2019 Conference Abstracts

03, funded by the Centers for Disease Control and Prevention. Its contents are solely the responsibility of the authors and do not necessarily represent the official view of the Centers for Disease Control and Prevention.

\section{References}

Rock PJ, Singleton MD. 2018. Assessing Definitions of Heroin Overdose in ED \& EMS Data Using Hospital Billing Data. Online J Public Health Inform. 10(1), e97. doi:10.5210/ojphi.v10i1.8695.

Vivolo-Kantor AM, Seth P, Gladden RM, Mattson CL, Baldwin GT, et al. (2016). Morbidity and Mortality Weekly Report Vital Signs: Trends in Emergency Department Visits for Suspected Opioid Overdoses — United States, 67(9), $279-285$.

Retrieved from https://www.cdc.gov/mmwr/volumes/67/wr/pdfs/mm6709e1-H.pdf

Figure 1

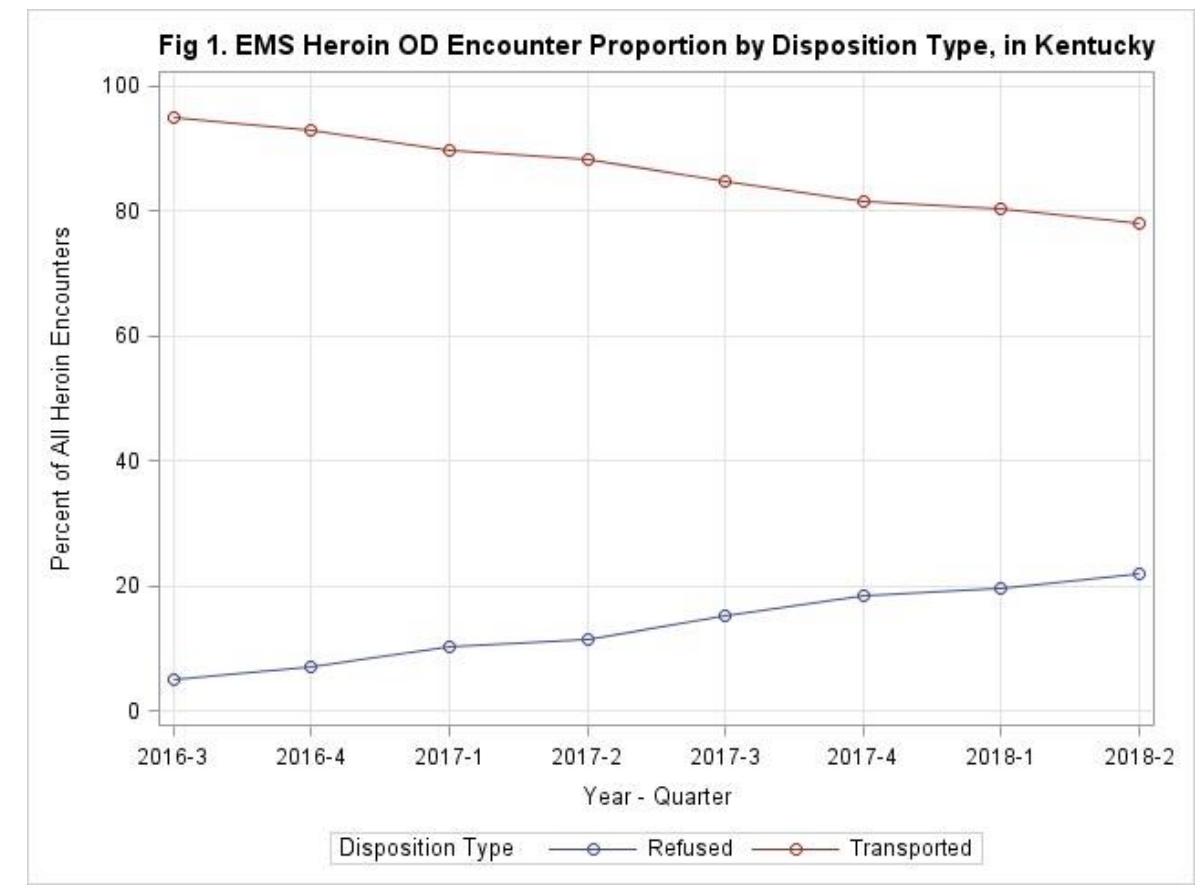

SDS Annual Conference Proceedings 2019. This is an Open Access article distributed under the terms of the Creative Commons AttributionNoncommercial 4.0 Unported License (http://creativecommons.org/licenses/by-nc/3.0/), permitting all non-commercial use, distribution, and reproduction in any medium, provided the original work is properly cited. 
Figure 2

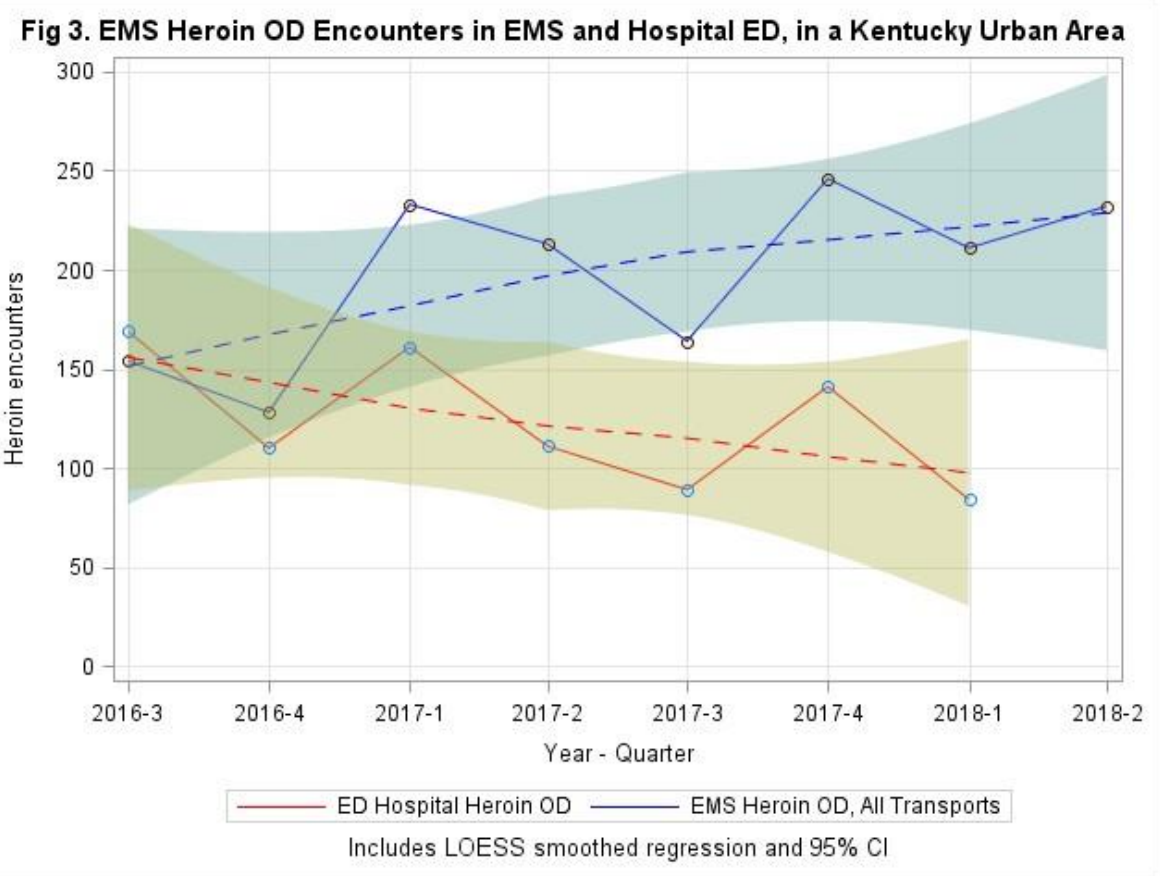

Figure 3

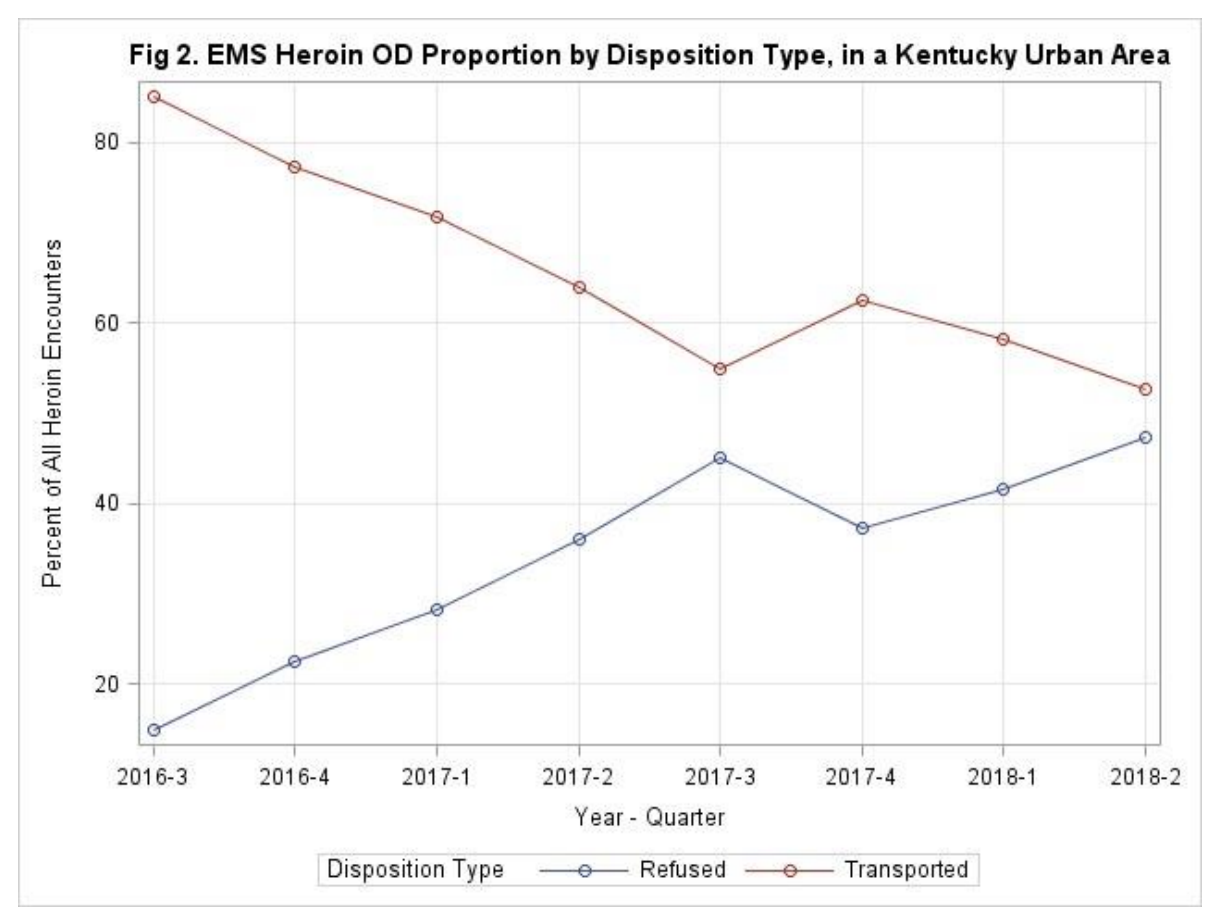

ISDS Annual Conference Proceedings 2019. This is an Open Access article distributed under the terms of the Creative Commons AttributionNoncommercial 4.0 Unported License (http://creativecommons.org/licenses/by-nc/3.0/), permitting all non-commercial use, distribution, and reproduction in any medium, provided the original work is properly cited. 